\title{
A GENERAL TYPE OF WEAK TRANSFORM
}

\section{Anthippi Poulkou}

Abstract. The existence of Fourier-type integrals of the form

$$
\int_{-\infty}^{\infty} h(a, x) e^{-a \theta(x)} d a
$$

as weak functions in the sense of Lighthill [9] and Jones [5] is established, when $\theta: \mathbb{R} \rightarrow \mathbb{R}$ is an infinitely differentiable function with everywhere non-vanishing first-order derivative, and the kernel function $h$ satisfies certain general conditions. Properties concerning the derivatives and the weak limits of the above weak functions are also obtained.

\section{Introduction and statements of results}

In mathematical physics, especially diffraction of radio waves, microwave problems, and optics, a large number of problems deal with the calculation of integrals of the type

$$
\iint_{S} j(x, y) e^{i k f(x, y)} d y d x
$$

where $j$ and $f$ are real functions and $k$ is real (see, for example, $[2,7]$ ). The integrals of the form (1.1) and also integral transforms, such as Hankel, Fourier, Laplace, Stieltjes, and Riemann-Liouville fractional integral transforms, can be viewed as

$$
\int_{0}^{\infty} g(x) k(a, x) d x
$$

where $g$ and the kernel $k$ are locally integrable functions on $(0, \infty)$ and $a$ is a positive parameter. To calculate (1.2), we often use asymptotic techniques [3,6], provided that, of course, the integral (1.2) exists in some ordinary sense. If the previous integral cannot be defined according to the classical theory of convergence, then it may be regarded as an integral transform of a distribution (generalized function); see [4] or [8]. Also, the consideration of (1.2) in a generalized sense makes the techniques which give asymptotic expansions of (1.2) as $a \rightarrow+\infty$ widely applicable. Mainly, there are two ways to extend the classical integral transforms to distributions.

The first one is used, for example, by Rudin [11], for the Fourier transform of tempered distributions and the second by L. Schwartz [12, p. 217], for the generalized Laplace transform.

In [4], Jones has given a detailed study of the asymptotic behaviour of (1.2) as $a \rightarrow+\infty$, defining it as a generalized function (in the terminology of Lighthill [9] and Jones [5]) when the kernel $k$ is an oscillatory function, such as $e^{i a x^{2}}$ and $g$ is a generalized function. The presence of a generalized function in the integrand not only

Received May 23, 1994. 
increases the range of validity of the analysis, but also simplifies the proofs in some aspects.

In an earlier paper [10], we have examined integrals of the type (1.2), where the kernel function $k$ belongs to a class wider than the one considered by Jones [4]. We have also defined (1.2) as weak functions, according to [5], so that the class of functions represented by (1.2) is larger than that of Jones' [4]. Another type of weak functions defined in [10] are integrals of the form

$$
\int_{-\infty}^{\infty} h(a, x) e^{-i a x} d a
$$

when the kernel $h$ belongs to a class

$$
H=\bigcup\{H(N, c, d): N \in \mathbb{R}, 0<c \leq 1,0 \leq d<1\}
$$

where $H(N, c, d)$ consists of all the infinitely differentiable functions, $h: \mathbb{R} \times \mathbb{R} \rightarrow \mathbb{R}$ with the property that for each $r, s=0,1,2, \cdots$ and each $a<b$, there exists a constant $C_{r, s, a, b}$ such that

$$
\left|\frac{\partial^{r+s}}{\partial x^{r} \partial a^{s}} h(a, x)\right| \leq C_{r, s, a, b}(1+|a|)^{N-c s+d r}
$$

for $a \in \mathbb{R}, x \in[a, b]$ (see Definition 3.1).

The theory of [10] is not sufficiently $\mathrm{c}$ nprehensive to embrace integrals of a more general nature, namely integrals of the $t$, se

$$
\int_{-\infty}^{\infty} h(a, x) e^{-i a \theta(x)} d a
$$

where $\theta$ is a smooth monotonic function and the kernel $h$ belongs to the same class $H$ as in [10], primarily because it rests so heavily upon the class $H$. Thus, a new definition has to be introduced. This involves a completely different approach from that of [10] and is carried out in this article. Of course, instead of (1.5) we can consider integrals by transforming to the inverse function $x(\theta)$ of $\theta(x)$, namely integrals of the form

$$
\int_{-\infty}^{\infty} j(a, \theta) e^{-i a \theta} d a
$$

But some caution is necessary, because the mapping may not be bounded and the range may be different from the domain.

The present paper deals with a problem suggested to us by Professor D. S. Jones. Its aim is to define integrals of the type (1.5) as weak functions and study some properties of them when $\theta$ is an infinitely differentiable function such that $\theta^{\prime}(x) \neq 0$, for $x \in \mathbb{R}$, and the kernel $h$ belongs to a class $H$ as in (1.4). Nevertheless, the class $H$ of kernel functions is wide and includes functions such as $e^{i\left(1+a^{2}\right)^{v} f}$ or $y\left(x\left(1+a^{2}\right)^{v}\right)$ with $y$ a good function (see Remark 3.2). In fact, we prove

Theorem 1. Let $h \in H, y$ a good function such that $y(0)=1$, and $\theta: \mathbb{R} \rightarrow \mathbb{R}$ an infinitely differentiable function such that $\theta^{\prime}(x) \neq 0$ for $x \in \mathbb{R}$. Then for $x \in \mathbb{R}$, the weak limit

$$
\lim _{\varepsilon \rightarrow 0} \int_{-\infty}^{\infty} y(\varepsilon a) h(a, x) e^{-i a \theta(x)} d a
$$


exists. This is denoted by

$$
\int_{-\infty}^{\infty} h(a, x) e^{-i a \theta(x)} d a
$$

Our analysis is carried out in the following way. At first we examine the existence of the $\lim _{\varepsilon \rightarrow 0} F(\varepsilon)$ where

$$
F(\varepsilon)=\int_{-\infty}^{\infty} \varphi(x) \int_{-\infty}^{\infty} y(\varepsilon a) h(a, x) e^{-i a \theta(x)} d a d x
$$

when $y, h$, and $\theta$ are as in Theorem 1 , and $\varphi$ is a fine function which vanishes outside $[a, b]$. Since the inner integral in (1.6) is bounded by

$$
\int_{-\infty}^{\infty}|y(\varepsilon a)|(1+|a|)^{N} d a
$$

we have that, so long as $N<-1, F(\varepsilon)$ is uniformly convergent on $[-p, p]$, for every $p>0$. It is desirable that this uniform convergence should bear no restriction on $N$ and consequently on the kernel function $h$. For this purpose we introduce a suitable first-order differential operator $M$ on the space of smooth functions (see Definition 3.4). Then the formula of the adjoint $K$ of $M$ is given (see Definition 3.5) and we show that the convergence of $F(\varepsilon)$ depends on the limit behaviour as $\varepsilon \rightarrow 0$ of the integral

$$
\int_{-\infty}^{\infty} \int_{-\infty}^{\infty} K\{\varphi(x) y(\varepsilon a) h(a, x)\} e^{-i a \theta x)} d a d x
$$

(see Proposition 3.6). Next, we prove that $K$ can be replaced by any term of a sequence $\left(P_{v}\right)$ of suitable operators, also defined on the space of smooth functions (see Proposition 3.13). The advantage of this fact is that, in this way, the integral (1.7) is split up into a finite number of integrals (see Definition 3.11 and Theorems 3.8 and $3.10)$ with simpler convergence properties. Now we are in a position to establish the convergence of the integral $F(\varepsilon)$ (see Theorem 4.1) and therefore give our main result.

From Theorem 1 the following two corollaries are derived. The first one deals with the weak derivative of the weak function defined in the above theorem and the second describes a property of the previously mentioned weak function.

Theorem 2. If $h \in H$ and $\theta: \mathbb{R} \rightarrow \mathbb{R}$ an infinitely differentiable function such that $\theta^{\prime}(x) \neq 0$ for every $x \in \mathbb{R}$, then

$$
\frac{d}{d x} \int_{-\infty}^{\infty} h(a, x) e^{-i a \theta(x)} d a=\int_{-\infty}^{\infty}\left\{\frac{\partial}{\partial x} h(a, x)-i a \theta^{\prime}(x) h(a, x)\right\} e^{-i a \theta(x)} d a
$$

for $x \in \mathbb{R}$, where the derivative on the left-hand side is a weak one.

Theorem 3. With the notation of Theorem 2, it holds that for every $x \in \mathbb{R}$

$$
\int_{-\infty}^{\infty} \frac{\partial}{\partial a} h(a, x) e^{-i a \theta(x)} d a=\int_{-\infty}^{\infty} i \theta(x) h(a, x) e^{-i a \theta(x)} d a .
$$

Finally, an interesting result is obtained (from Theorem 4.1). This concerns the weak limit of a sequence of weak functions of the same type as in Theorem 1. 
Theorem 4. Let $\theta: \mathbb{R} \rightarrow \mathbb{R}$ be an infinitely differentiable function with $\theta^{\prime}(x) \neq 0$ for every $x \in \mathbb{R}$, and $\left(h_{n}\right)$ a sequence such that $h_{n}: \mathbb{R} \times \mathbb{R} \rightarrow \mathbb{R}$ and the following three conditions hold:

(i) $h_{n} \in H(N, c, d)$ for every $n \in \mathbb{N}$;

(ii) there exist $h \in H(N, c, d)$ and $a_{0} \in \mathbb{R}$ such that

$$
\lim _{n \rightarrow \infty} h_{n}\left(a_{0}, x\right)=h\left(a_{0}, x\right)
$$

for every $x \in \mathbb{R}$;

(iii) for every $p \in \mathbb{N}$ the sequence $\left(\frac{\partial^{p}}{\partial a^{p}} h_{n}\right)$ as $n \rightarrow \infty$ converges uniformly on $\mathbb{R}$. Then

$$
\lim _{n \rightarrow \infty} \int_{-\infty}^{\infty} h_{n}(a, x) e^{-i a \theta(x)} d a=\int_{-\infty}^{\infty} h(a, x) e^{-i a \theta(x)} d a .
$$

\section{Preliminaries and definitions}

In this section, we introduce the notation and terminology that we use throughout this paper. We note that the definitions and propositions we present below can be found in [5].

A fine function is an infinitely differentiable function which, together with all its derivatives, vanishes outside a finite interval. Every fine function is bounded.

A sequence of fine functions $\left(\varphi_{n}\right)$ is called $f$-regular if, for every fine $\varphi$,

$$
\lim _{n \rightarrow \infty} \int_{-\infty}^{\infty} \varphi_{n}(x) \varphi(x) d x
$$

exists (and is finite). Two $f$-regular sequences $\left(\varphi_{n}\right)$ and $\left(\psi_{n}\right)$ are called $f$-equivalent if

$$
\lim _{n \rightarrow \infty} \int_{-\infty}^{\infty} \varphi_{n}(x) \varphi(x) d x=\lim _{n \rightarrow \infty} \int_{-\infty}^{\infty} \psi_{n}(x) \varphi(x) d x
$$

for every fine $\varphi$. An $f$-equivalence class of $f$-regular sequences is called a weak function. If $w$ is a weak function and $\left(\varphi_{n}\right)$ an $f$-regular sequence that belongs to $w$, then we say that $\left(\varphi_{n}\right)$ defines $w$ and we write

$$
\int_{-\infty}^{\infty} w(x) \varphi(x) d x=\lim _{n \rightarrow \infty} \int_{-\infty}^{\infty} \varphi_{n}(x) \varphi(x) d x
$$

where $\varphi$ is fine.

If the $f$-regular sequence $\left(\varphi_{n}\right)$ defines the weak function $w$, then the sequence $\left(\varphi_{n}^{\prime}\right)$ is also $f$-regular and defines a weak function which is denoted by $w^{\prime}$ and it is called the weak derivative of $w$.

If $\left(w_{n}\right)$ is a sequence of weak functions and $w$ a weak function, then we say that the weak limit of $\left(w_{n}\right)$

exists and is equal to $w$ if

$$
\lim _{n \rightarrow \infty} w_{n}
$$

$$
\lim _{n \rightarrow \infty} \int_{-\infty}^{\infty} w_{n}(x) \varphi(x) d x=\int_{-\infty}^{\infty} w(x) \varphi(x) d x
$$

for every fine $\varphi$.

Analogous is the definition of $\lim _{\mu \rightarrow \mu_{0}} w_{\mu}$, where $\mu$ is a parameter which runs through the points of an interval of $\mathbb{R}$ and $w_{\mu}$ is a weak function for each value of $\mu$. 
The space of weak functions is complete under weak limits (cf. [5, p. 447]).

A function $y: \mathbb{R} \rightarrow \mathbb{C}$ is called good (cf. also [9]) if it is infinitely differentiable and

$$
\lim _{|x| \rightarrow \infty}\left|x^{r} y^{(k)}(x)\right|=0
$$

for any integers $r, k \geq 0$, where $y^{(k)}(x)=\frac{d^{k}}{d x^{k}} y(x)$ and this notation for the $k$ th derivative will be used throughout the paper.

Analogous to the definition of the weak function is that of the generalized function which is defined as an equivalence class of regular sequences of good functions.

Any generalized function is necessarily a weak function but the converse is not true.

Remark 2.1. There is complete equivalence between the meaning of weak functions and the meaning of distributions (defined by means of linear functionals of fine functions) given by L. Schwartz in [13]. For more details we refer to [5, p. 495].

The definition of generalized functions given by L. Schwartz in [13] uses a different approach. The space of good functions is denoted by $\mathcal{G}$. The dual $\mathcal{G}^{\prime}$ of $\mathcal{G}$ is the space of generalized functions which are called in [13] tempered distributions or distributions of slow growth. For a detailed explanation we refer to [5, p. 58].

\section{Kernel functions and operators}

In this section, we define the class $H$, where the kernel function $h$ belongs (in (3.1)) and we give some examples of elements of $H$ (in (3.2)). Then we introduce the operator $M$ (in (3.4)), the formula of the adjoint $K$ of $M$ (in (3.5)), and by means of $K$ and $M$ we get the form that the Fourier-type integrals can take (in (3.6)). Finally, we show that $K$ can be replaced by any term of a sequence $\left(P_{v}\right)$ of suitable operators (in (3.13)). Throughout our analysis, it will be assumed that $a$ and $x$ are real.

Definition 3.1. Let $N \in \mathbb{R}, 0<c \leq 1,0 \leq d<1$. The class $H(N, c, d)$ consists of all functions $h: \mathbb{R} \times \mathbb{R} \rightarrow \mathbb{R}$ such that

(i) $h$ is infinitely differentiable;

(ii) for each $r, s=0,1,2, \ldots$, and each $a<b$, there exists a constant $C_{r, s, a, b}$ such that

$$
\left|\frac{\partial^{r+s}}{\partial x^{r} \partial a^{s}} h(a, x)\right| \leq C_{r, s, a, b}(1+|a|)^{N-c s+d r}
$$

for $a \in \mathbb{R}, x \in[a, b]$.

Finally we write $H=\bigcup\{H(N, c, d): N \in \mathbb{R}, 0<c \leq 1,0 \leq d<1\}$.

Remark 3.2. Some examples of functions which are in $H$ (for proper constants $N, c, d)$ are given below (see also [10]), in a way that the class $H$ ends up to be a quite rich class of functions. Consequently we have

\begin{tabular}{|l|l|l|}
\hline 1. & $h(a, x)=\left\{\left(1+a^{2}\right)^{1 / 2}+\left(l+x^{2}\right)^{1 / 2}\right\}^{-1}$ & $H(-1,1,0)$ \\
\hline 2. & $h(a, x)=e^{i\left(1+a^{2}\right)^{1 / 4} x}$ & $H\left(0, \frac{1}{2}, \frac{1}{2}\right)$ \\
\hline 3. & $h(a, x)=e^{i\left(1+a^{2}\right)^{v} f(x) \quad\left(f: \mathbb{R} \rightarrow \mathbb{R}, \text { smooth, } 0 \leq v<\frac{1}{2}\right)}$ & $H(0,1-2 v, 2 v)$ \\
\hline 4. & $h(a, x)=y\left\{x\left(1+a^{2}\right)^{v}\right\} \quad\left(y\right.$ good, $\left.0 \leq v<\frac{1}{2}\right)$ & $H(0,1-2 v, 2 v)$ \\
\hline
\end{tabular}

It should be noted that all the above examples of $h$ can be handled similarly by induction. 
The following Proposition enables us to define our operators.

Proposition 3.3. If $n$ is a fixed positive number and $\chi: \mathbb{R} \rightarrow \mathbb{R}$ a fine function such that $\chi(a)=1$ for $a \in[-n, n]$, then for every $k, s=0,1,2, \ldots$, there exists a constant $C$ such that

$$
\left|\frac{d^{k}}{d a^{k}}\left(\frac{1-\chi(a)}{a}\right)^{s}\right| \leq C(1+|a|)^{-(s+k)}
$$

for every $a \in \mathbb{R}$.

Proof. We use induction and the following arguments. There exists $p>0$ such that $\chi(a)=0$ for $|a| \geq n+p$. Hence, if $(1-\chi(a)) / a=\chi_{1}(a)$, we have

$$
\begin{array}{ll}
\left|\chi_{1}(a)\right|=0, & \text { for }|a| \leq n, \\
\left|\chi_{1}(a)\right| \leq \frac{1+|\chi(a)|}{|a|}=C_{1}, & \text { for } n<|a|<n+p \\
\left|\chi_{1}(a)\right|=\frac{1}{|a|}, & \text { for }|a| \geq n+p .
\end{array}
$$

With the above information at our disposal we give

Definition 3.4. Let $\theta: \mathbb{R} \rightarrow \mathbb{R}$ be a function such that (i) $\theta$ is infinitely differentiable and (ii) $\theta^{\prime}(x) \neq 0$ for every $x \in \mathbb{R}, n$ be a fixed positive number and $\chi: \mathbb{R} \rightarrow \mathbb{R}$ a fine function such that $\chi(a)=1$ for $-n \leq a \leq n$. We set

$$
\{M(\varphi)\}(a, x)=\frac{i\{1-\chi(a)\}\left\{a^{2} \theta(x) \frac{\partial}{\partial a} \varphi(a, x)+a \theta^{\prime}(x) \frac{\partial}{\partial x} \varphi(a, x)\right\}}{a^{2}\left\{\theta^{2}(x)+\theta^{\prime 2}(x)\right\}}+\chi(a) \varphi(a, x),
$$

for $(a, x) \in \mathbb{R} \times \mathbb{R}$ and $\varphi: \mathbb{R} \times \mathbb{R} \rightarrow \mathbb{R}$ an infinitely differentiable function.

Definition 3.5. Let $\theta, \chi, \varphi$ be as in Definition 3.4. We set for $(a, x) \in \mathbb{R} \times \mathbb{R}$,

$$
\begin{aligned}
& \left\{K_{1}(\varphi)\right\}(a, x)=-i \frac{\theta(x)}{\theta^{2}(x)+\theta^{\prime 2}(x)} \frac{\partial}{\partial a} \varphi(a, x), \\
& \left\{K_{2}(\varphi)\right\}(a, x)=-i \frac{1-\chi(a)}{a} \frac{\partial}{\partial x}\left\{\frac{\theta^{\prime}(x)}{\theta^{2}(x)+\theta^{\prime 2}(x)} \varphi(a, x)\right\}, \\
& \left\{K_{3}(\varphi)\right\}(a, x)=\left\{1-\frac{\theta^{2}(x)}{\theta^{2}(x)+{\theta^{\prime}}^{2}(x)}\right\} \chi(a) \varphi(a, x), \\
& \{K(\varphi)\}(a, x)=\left\{K_{1}(\varphi)\right\}(a, x)+\left\{K_{2}(\varphi)\right\}(a, x)+\left\{K_{3}(\varphi)\right\}(a, x) .
\end{aligned}
$$

The connection between the above defined operators $M$ and $K$ as well as a property of $M$ are demonstrated by the following result.

Proposition 3.6. If $h \in H, \varphi$ is a fine function, $\gamma$ a good function and $\theta: \mathbb{R} \rightarrow \mathbb{R}$ an infinitely differentiable function such that $\theta^{\prime}(x) \neq 0$ for every $x \in \mathbb{R}$, we set

$$
F=\int_{-\infty}^{\infty} \int_{-\infty}^{\infty} \varphi(x) \gamma(\varepsilon a) h(a, x) e^{-i a \theta(x)} d a d x
$$


for all finite $\varepsilon \in \mathbb{R}$. Then

$$
\begin{aligned}
F & =\int_{-\infty}^{\infty} \int_{-\infty}^{\infty} \varphi(x) \gamma(\varepsilon a) h(a, x) M\left\{e^{-i a \theta(x)}\right\} d a d x \\
& =\int_{-\infty}^{\infty} \int_{-\infty}^{\infty} K\{\varphi(x) \gamma(\varepsilon a) h(a, x)\} e^{-i a \theta(x)} d a d x
\end{aligned}
$$

(where $M$ and $K$ are defined in (3.4) and (3.5) respectively).

Proof. The first wanted equality is obtained by Definition 3.4 and also

$$
F=I+J+\int_{-\infty}^{\infty} \int_{-\infty}^{\infty}\left\{1-\frac{\theta^{2}(x)}{\theta^{2}(x)+{\theta^{\prime}}^{2}(x)}\right\} \chi(a) \varphi(x) \gamma(\varepsilon a) h(a, x) e^{-i a \theta(x)} d a d x
$$

where

$$
I=i \int_{-\infty}^{\infty} \frac{\theta(x)}{\theta^{2}(x)+{\theta^{\prime}}^{2}(x)} \varphi(x) \int_{-\infty}^{\infty} \gamma(\varepsilon a) h(a, x) \frac{\partial}{\partial a} e^{-i a \theta(x)} d a d x
$$

and

$$
J=i \int_{-\infty}^{\infty} \frac{\left.\theta^{\prime} x\right)}{\theta^{2}(x)+{\theta^{\prime}}^{2}(x)} \varphi(x) \int_{-\infty}^{\infty} \frac{1-\chi(a)}{a} \gamma(\varepsilon a) h(a, x) \frac{\partial}{\partial x} e^{-i a \theta(x)} d a d x .
$$

After an integration by parts $J$ can be written as

$$
\begin{aligned}
J= & -i \int_{-\infty}^{\infty}\left\{\frac{\theta^{\prime}(x)}{\theta^{2}(x)+\theta^{\prime 2}(x)} \varphi(x)\right\}^{\prime} \int_{-\infty}^{\infty} \frac{1-\chi(a)}{a} \gamma(\varepsilon a) h(a, x) e^{-i a \theta(x)} d a d x \\
& -i \int_{-\infty}^{\infty} \frac{\theta^{\prime}(x)}{\theta^{2}(x)+\theta^{\prime 2}(x)} \varphi(x) \int_{-\infty}^{\infty} \frac{1-\chi(a)}{a} \gamma(\varepsilon a) \frac{\partial}{\partial x} h(a, x) e^{-i a \theta(x)} d a d x,
\end{aligned}
$$

since the function $\left(\theta^{\prime}(x) /\left(\theta^{2}(x)+{\theta^{\prime}}^{2}(x)\right)\right) \varphi(x)$ is fine and the integral

$$
\int_{-\infty}^{\infty} \frac{1-\chi(a)}{a} \gamma(\varepsilon a) h(a, x) e^{-i a \theta(x)} d a
$$

finite (for the latter see Proposition 3.3 and note that $\gamma$ is good and $h \in H$ ). Therefore from (3.1), because of (3.2) and an integration by parts of the inner integral of $I$, the conclusion follows.

It is easily understood along the lines of the proof of the above proposition that if, in $F$, we apply the operator $M$ on $e^{-i a \theta(x)}$ for a second time and use the same procedure as before, we shall obtain the operator $K$ applied on $K(\varphi \gamma h)$. So, by a repetition of the process, we can get an idea as to the form the integral $F$ may take. Thus, we now have the following definition.

Definition 3.7. Let $\psi: \mathbb{R} \times \mathbb{R} \rightarrow \mathbb{R}$ be an infinitely differentiable function and $v=$ $1,2, \ldots$ We set for $(a, x) \in \mathbb{R} \times \mathbb{R}$,

$$
\left\{L_{v}(\psi)\right\}(a, x)=\left\{\left\{K_{1}(\psi)+K_{2}(\psi)\right\}^{v}\right\}(a, x)
$$

where $K_{1}$ and $K_{2}$ are as in Definition 3.5. 
Theorem 3.8. With the notation of Definition 3.7, it holds that, for all integers $\tau$, $\mu, j$ with

$$
0 \leq \tau \leq v-1, \quad 1 \leq \mu \leq v-\tau, \quad 1 \leq j \leq \mu
$$

there exist constants ${ }^{v} b_{\tau, \mu, j},{ }^{v} c_{\tau, \mu}$ such that

$$
L_{v}(\psi)={ }^{v} A(\psi)+\sum_{\tau, \mu, j}{ }^{v} b_{\tau, \mu, j}{ }^{v} B_{\tau, \mu, j}(\psi)+\sum_{\tau, \mu}{ }^{v} c_{\tau, \mu}{ }^{v} C_{\tau, \mu}(\psi)
$$

where

$$
\begin{gathered}
\left\{{ }^{v} A(\psi)\right\}(a, x)=(-i)^{v} \Theta^{v}(x) \frac{\partial^{v}}{\partial a^{v}} \psi(a, x), \\
\left\{{ }^{v} B_{\tau, \mu, j}(\psi)\right\}(a, x)=(-i)^{v}\left\{\left\{\frac{1-\chi(a)}{a}\right\}^{\mu}\right\}^{(v-\tau-\mu)} \\
\times\left\{\Theta_{1}^{\mu}(x) \Theta^{v-\mu}(x)\right\}^{(\mu-j)} \frac{\partial^{\tau+j}}{\partial a^{\tau} \partial x^{j}} \psi(a, x), \\
\left\{{ }^{v} C_{\tau, \mu}(\psi)\right\}(a, x)=(-i)^{v}\left\{\left\{\frac{1-\chi(a)}{a}\right\}^{\mu}\right\}^{(v-\tau-\mu)} \\
\times\left\{\Theta^{v-\tau-\mu}(x)\left\{\Theta_{1}^{\mu}(x) \Theta^{\tau}(x)\right\}^{\prime}\right\}^{(\mu-1)} \frac{\partial^{\tau}}{\partial a^{\tau}} \psi(a, x),
\end{gathered}
$$

and

$$
\Theta(x)=\frac{\theta(x)}{\theta^{2}(x)+{\theta^{\prime}}^{2}(x)}, \quad \Theta_{1}(x)=\frac{\theta^{\prime}(x)}{\theta^{2}(x)+{\theta^{\prime}}^{2}(x)}
$$

Proof. We use induction. It is obvious that for $v=1$, (3.4) holds with ${ }^{1} b_{0,1,1}={ }^{1} c_{0,1}=$ 1. The proof of the next step of the induction is a matter of routine by using simple differentiation rules.

We give now the following definition, which will be useful in our calculations.

Definition 3.9. Let $\psi: \mathbb{R} \times \mathbb{R} \rightarrow \mathbb{R}$ be an infinitely differentiable function and $v=$ $1,2, \cdots$. For $(a, x) \in \mathbb{R} \times \mathbb{R}$, we set

$$
\left\{M_{v}(\psi)\right\}(a, x)=\left\{K_{3} \sum_{i=0}^{v-1}\left\{K_{1}(\psi)+K_{2}(\psi)\right\}^{i}\right\}(a, x)
$$

where $K_{1}, K_{2}$, and $K_{3}$ are defined in (3.5).

A consequence of the preceding definition and of Theorem 3.8 is

Theorem 3.10. With the notation of Definition 3.9, it holds that for all integers $\sigma, \rho, q, \lambda$ with

$$
0 \leq \sigma \leq \lambda-2, \quad 1 \leq \rho \leq \lambda-1-\sigma, \quad 1 \leq q \leq \rho, \quad 2 \leq \lambda \leq v
$$


there exist constants ${ }^{\lambda} b_{\sigma, \rho, q},{ }^{\lambda} c_{\sigma, \rho}$ such that

$$
\begin{aligned}
M_{v}(\varphi)=\chi(1-\theta \Theta) & \sum_{k=0}^{v-1}{ }^{k} A(\psi)+(1-p) \chi(1-\theta \Theta) \\
& \times \sum_{\lambda=2}^{v}(-i)^{\lambda-1}\left\{\sum_{\sigma, \rho, q}{ }^{\lambda} b_{\sigma, \rho, q}{ }^{\lambda-1} B_{\sigma, \rho, q}(\psi)+\sum_{\sigma, \rho}{ }^{\lambda} c_{\sigma, \rho}{ }^{\lambda-1} C_{\sigma, \rho}(\psi)\right\},
\end{aligned}
$$

where $p=1$ for $v=1$ and $p=0$ for $v \geq 2$ and $\Theta,{ }^{k} A,{ }^{\lambda-1} B_{\sigma, \rho, q},{ }^{\lambda-1} C_{\sigma, \rho}$ are defined in the same way as in (3.5).

Proof. We use induction on $v$ and we can easily see that the theorem holds from the very definitions.

We are now in a position to give the following definition.

Definition 3.11. Let $\psi: \mathbb{R} \times \mathbb{R} \rightarrow \mathbb{R}$ be an infinitely differentiable function and $v=$ $1,2, \cdots$. We set for $(a, x) \in \mathbb{R} \times \mathbb{R}$,

$$
\left\{P_{v}(\psi)\right\}(a, x)=\left\{L_{v}(\psi)+M_{v}(\psi)\right\}(a, x)
$$

where $L_{v}$ and $M_{v}$ are defined in Definitions 3.7 and 3.9, respectively.

We shall need the following lemma.

Lemma 3.12. Let $L_{v}$ be as in Definition 3.7. For every $v=1,2, \ldots$, we have that

$$
L_{v}\{\varphi(x) \gamma(\varepsilon a) h(a, x)\}=\sum_{j, \lambda, \sigma} \varphi_{j}(x) \gamma_{\lambda}(\varepsilon a) h_{\sigma}(a, x)
$$

where the integers $j, \lambda, \sigma=0, \ldots, v$ and $\varphi, \varphi_{j}$ are fine functions, $\gamma, \gamma_{\lambda}$ are good functions, and $h, h_{\sigma} \in H$.

Proof. This is an immediate result of Theorem 3.8 by using Leibniz's Theorem and Proposition 3.3. (We have also used the substitution $\varepsilon a=\rho$.)

A consequence of the above lemma is the following proposition of which the proof, done by induction and being trivial, is omitted.

Proposition 3.13. If $F$ is as in Proposition 3.6 and $P_{v}$ as in Definition 3.11, then for every $v=1,2, \ldots$, it holds that

$$
F=\int_{-\infty}^{\infty} \int_{-\infty}^{\infty} P_{v}\{\varphi(x) \gamma(\varepsilon a) h(a, x)\} e^{-i a \theta(x)} d a d x
$$

The following remark is proved by Proposition 3.13. 
Remark 3.14. Let $F$ be as in Proposition 3.6. Then for every $v=1,2, \ldots$, we obtain

$$
\begin{aligned}
F=( & -i)^{v} I_{0}+(-i)^{v} \sum_{\tau, \mu, j} \sum_{i=0}^{j}\left(\begin{array}{l}
j \\
i
\end{array}\right)^{v} b_{\tau, \mu, j} I_{1}+(-i)^{v} \sum_{\tau, \mu}{ }^{v} c_{\tau, \mu} I_{2}+\sum_{k=0}^{v-1}(-i)^{k} I_{3} \\
& +(1-p) \sum_{\lambda=2}^{v} \sum_{\sigma, \rho, q} \sum_{m=0}^{q}(-i)^{\lambda-1}\left(\begin{array}{c}
q \\
m
\end{array}\right){ }^{\lambda} b_{\sigma, \rho, q} I_{4} \\
& +(1-p) \sum_{\lambda=2}^{v} \sum_{\sigma, \rho}(-i)^{\lambda-1}{ }^{\lambda} c_{\sigma, \rho} I_{5},
\end{aligned}
$$

with the integers $\tau, \mu$, and $j$ satisfying (3.3) and $\sigma, \rho, q$ as in (3.6), $p=1$ for $v=1$, $p=0$ for $v \geq 2$, and

$$
\begin{aligned}
& I_{0}=\int_{-\infty}^{\infty} \Theta^{v}(x) \varphi(x) \int_{-\infty}^{\infty} \frac{\partial^{v}}{\partial a^{v}}\{\gamma(\varepsilon a) h(a, x)\} e^{-i a \theta(x)} d a d x \\
& I_{1}=\int_{-\infty}^{\infty}\left\{\Theta_{1}^{\mu}(x) \Theta^{v-\mu}(x)\right\}^{(\mu-j)} \varphi^{(i)}(x) \\
& \times \int_{-\infty}^{\infty}\left\{\left\{\frac{1-\chi(a)}{a}\right\}^{\mu}\right\}^{(v-\tau-\mu)} \frac{\partial^{\tau+j-1}}{\partial a^{\tau} \partial x^{j-i}}\{\gamma(\varepsilon a) h(a, x)\} e^{-i a \theta(x)} d a d x, \\
& I_{2}=\int_{-\infty}^{\infty}\left\{\Theta^{v-\tau-\mu}(x)\left\{\Theta_{1}^{\mu}(x) \Theta^{\tau}(x)\right\}^{\prime}\right\}^{(\mu-1)} \varphi(x) \\
& \times \int_{-\infty}^{\infty}\left\{\left\{\frac{1-\chi(a)}{a}\right\}^{\mu}\right\}^{(v-\tau-\mu)} \frac{\partial^{\tau}}{\partial a^{\tau}}\{\gamma(\varepsilon a) h(a, x)\} e^{-i a \theta(x)} d a d x \\
& I_{3}=\int_{-\infty}^{\infty}\{1-\theta(x) \Theta(x)\} \Theta^{k}(x) \varphi(x) \int_{-\infty}^{\infty} \chi(a) \frac{\partial^{k}}{\partial a^{k}}\{\gamma(\varepsilon a) h(a, x)\} e^{-i a \theta(x)} d a d x \\
& I_{4}=\int_{-\infty}^{\infty}\{1-\theta(x) \Theta(x)\}\left\{\Theta^{\rho}(x) \Theta^{\lambda-1-\rho}(x)\right\}^{(\rho-q)} \varphi^{(m)}(x) \\
& \times \int_{-\infty}^{\infty} \chi(a)\left\{\left\{\frac{1-\chi(a)}{a}\right\}^{\rho}\right\}^{(\lambda-1-\sigma-\rho)} \frac{\partial^{\sigma+q-m}}{\partial a^{\sigma} \partial x^{q-m}}\{\gamma(\varepsilon a) h(a, x)\} e^{-i a \theta(x)} d a d x, \\
& I_{5}=\int_{-\infty}^{\infty}\{1-\theta(x) \Theta(x)\}\left\{\Theta^{\lambda-1-\sigma-\rho}(x)\left\{\Theta_{1}^{\rho}(x) \Theta^{\sigma}(x)\right\}^{\prime}\right\}^{(\rho-1)} \varphi(x) \\
& \times \int_{-\infty}^{\infty} \chi(a)\left\{\left\{\frac{1-\chi(a)}{a}\right\}^{\rho}\right\}^{(\lambda-1-\sigma-\rho)} \frac{\partial^{\sigma}}{\partial a^{\sigma}}\{\gamma(\varepsilon a) h(a, x)\} e^{-i a \theta(x)} d a d x .
\end{aligned}
$$

\section{The weak transform}

The kernel function $h(a, x)$ introduced in the preceding section, as well as the use of operators defined above, enable us to investigate the uniform convergence of the integral $F$ of Proposition 3.6 and therefore prove our main result (Theorem 1) which defines the considered Fourier-type integrals as weak functions. So, we can show the following result. 
Theorem 4.1. If $h \in H, \varphi$ is a fine function, $\gamma$ a good function, and $\theta: \mathbb{R} \rightarrow \mathbb{R}$ an infinitely differentiable function such that $\theta^{\prime}(x) \neq 0$ for every $x \in \mathbb{R}$, then the integral

$$
F(\varepsilon)=\int_{-\infty}^{\infty} \varphi(x) \int_{-\infty}^{\infty} \gamma(\varepsilon a) h(a, x) e^{-i a \theta(x)} d a d x
$$

converges uniformly on the interval $[-p, p]$ for every $p>0$.

Proof. By Remark 3.14, from the type of the integrand of the integrals $I_{3}, I_{4}$, and $I_{5}$, we observe that these can be written as

$$
\int_{-\infty}^{\infty} \varphi_{0}(x) \int_{-\infty}^{\infty} \chi(a) \psi_{\varepsilon}(a, x) e^{-i a \theta(x)} d a d x
$$

where $\varphi_{0}, \chi$ are fine functions and $\psi_{\varepsilon}$ is an infinitely differentiable function. Therefore the integrals $I_{3}(\varepsilon), I_{4}(\varepsilon), I_{5}(\varepsilon)$ converge uniformly on the interval $[-p, p]$ for every $p>0$, since both their inner and outer integrals are, in fact, over finite intervals.

We consider the following cases for $h \in H(N, c, d)$ :

(i) $c+d \leq 1$. By the definition of $I_{0}$ (see Remark 3.14) and Definition 3.1, we obtain

$$
I_{0}(\varepsilon)=v F_{1}(\varepsilon)+\sum_{j=1}^{v}\left(\begin{array}{l}
v \\
j
\end{array}\right) F_{2}(\varepsilon)
$$

where

$$
\begin{gathered}
F_{1}(\varepsilon)=\int_{-\infty}^{\infty} \varphi_{1}(x) \int_{-\infty}^{\infty} \gamma(\varepsilon a) h_{v}(a, x) e^{-i a \theta(x)} d a d x \\
F_{2}(\varepsilon)=\int_{-\infty}^{\infty} \varphi_{1}(x) \int_{-\infty}^{\infty} \frac{\partial^{j}}{\partial a^{j}} \gamma(\varepsilon a) h_{v-j}(a, x) e^{-i a \theta(x)} d a d x,
\end{gathered}
$$

and by $\varphi_{1}$ we denote the fine function $\Theta^{v} \varphi$ such that $\varphi_{1}(x)=0$ for every $x \notin[a, b]$, with $[a, b]$ the interval for which (ii) of Definition 3.1 holds and also $h_{v} \in H(N-c v, c, d)$, and $h_{v-j} \in H(N-c(v-j), c, d), j=1,2, \ldots, v$. Similarly, and by using Proposition 3.3 for the integrals $I_{1}$ and $I_{2}$, we get

$$
\begin{gathered}
I_{1}(\varepsilon)=I_{2}(\varepsilon)=F_{1}(\varepsilon) \quad(\text { for } \tau=0), \\
I_{1}(\varepsilon)=I_{2}(\varepsilon)=F_{1}(\varepsilon)+\sum_{k=1}^{\tau}\left(\begin{array}{l}
\tau \\
k
\end{array}\right) F_{2}(\varepsilon) \quad(\text { for } 1 \leq \tau \leq v-1) .
\end{gathered}
$$

But from (4.3), for the inner integral of $F_{1}(\varepsilon)$, it holds that for every $a<b$ there exists a constant $C$ such that

$$
\left|\int_{-\infty}^{\infty} \gamma(\varepsilon a) h_{v}(a, x) e^{-i a \theta(\chi)} d a\right| \leq C \int_{-\infty}^{\infty}|\gamma(\varepsilon a)|(1+|a|)^{N-c v} d a
$$

for every $x \in[a, b], a \in \mathbb{R}$. For a given $N$ we can choose $v$ so that $N-c v<-1$. Hence the integral on the right of the above inequality is finite, since $\gamma$, being good, is bounded [cf. 5]. Therefore the inner integral of $F_{1}(\varepsilon)$ converges uniformly on the interval $[-p, p]$ for every $p>0$. The same holds for the inner integral of $F_{2}(\varepsilon)$ because it can be bounded by $C_{0} \int_{-\infty}^{\infty}(|\varepsilon|+|y|)^{j-1} \gamma^{(j)}(y) d y$, where $y=\varepsilon a$. Hence $F_{1}(\varepsilon), F_{2}(\varepsilon)$, and consequently (by (4.2) and (4.4)) $I_{0}(\varepsilon), I_{1}(\varepsilon)$, and $I_{2}(\varepsilon)$ are uniformly convergent on $[-p, p]$ for every $p>0$. 
(ii) $c+d>1$. Similarly to the above by replacing $c$ by $1-d$.

Gathering our conclusions, we have that the integral $F(\varepsilon)$ is split up into a finite number of integrals which are uniformly convergent on the interval $[-p, p]$ for every $p>0$ and every $h \in H$. The proof of the theorem is complete.

We now have all the pieces of information that are necessary to prove the main result of this paper.

Theorem 1. Let $h \in H, \gamma$ a good function such that $\gamma(0)=1$, and $\theta: \mathbb{R} \rightarrow \mathbb{R}$ an infinitely differentiable function with $\theta^{\prime}(x) \neq 0$ for $x \in \mathbb{R}$. Then for $x \in \mathbb{R}$, the weak limit

$$
\lim _{\varepsilon \rightarrow 0} \int_{-\infty}^{\infty} \gamma(\varepsilon a) h(a, x) e^{-i a \theta(x)} d a
$$

exists. This is denoted by

$$
\int_{-\infty}^{\infty} h(a, x) e^{-i a \theta(x)} d a
$$

Proof. From Remark 3.14 and using the same calculations as for the integrals $I_{0}, I_{1}$, and $I_{2}$ in the proof of Theorem 4.1, we observe that the integrals $I_{3}, I_{4}$, and $I_{5}$ are of the form

$$
\begin{gathered}
I(\varepsilon)=\sum_{k=0}^{v-1} \int_{-\infty}^{\infty} \varphi(x) \int_{-\infty}^{\infty} \chi(a) \gamma(\varepsilon a) h_{k}(a, x) e^{-i a \theta(x)} d a d x \\
J(\varepsilon)=\sum_{k=0}^{v-1} \int_{-\infty}^{\infty} \varphi(x) \int_{-\infty}^{\infty} \chi(a) \frac{\partial^{\rho}}{\partial a^{\rho}} \gamma(\varepsilon a) h_{k-\rho}(a, x) e^{-i a \theta(x)} d a d x
\end{gathered}
$$

where $\rho=1, \ldots, k, h_{i} \in H(N-c i, c, d)$ for $i=k, k-\rho$. Therefore the integral $F(\varepsilon)$ given by (4.1) is split up into a finite number of integrals of the form $F_{1}(\varepsilon)$, $F_{2}(\varepsilon)$ and $I(\varepsilon), J(\varepsilon)$, given by (4.3) and (4.5), respectively, which are continuous functions on the interval $[-p, p]$ for every $p>0$, because of their uniform convergence. The limits of $F_{1}(\varepsilon)$ and $I(\varepsilon)$ as $\varepsilon \rightarrow 0$ are obtained by replacing $\gamma(\varepsilon a)$ by 1 in their integrands. Also, for the integrals $F_{2}(\varepsilon)$ and $J(\varepsilon)$, we make the substitution $y=\varepsilon a$ which gives $\frac{\partial^{k}}{\partial a^{k}} \gamma(\varepsilon a)=\varepsilon^{k} \gamma^{(k)}(y), k=1,2, \ldots, v$, and we consider $\varepsilon^{k}$ outside the two signs of integration. Then the inner integrals of $F_{2}(\varepsilon)$ and $J(\varepsilon)$ are finite (since for that of $F_{2}(\varepsilon)$ we have chosen suitable $v$ ) and their outer integrals are independent of $\varepsilon$. Therefore the limits of $F_{2}(\varepsilon)$ and $J(\varepsilon)$ as $\varepsilon \rightarrow 0$ are equal to zero. Consequently, we arrive at

$$
\lim _{\varepsilon \rightarrow 0} F(\varepsilon)=G
$$

where $G$ is the right-hand side of (3.7) with 1 instead of $\gamma(\varepsilon a)$ (in the integrands of $I_{0}$ up to $I_{5}$ ). Hence (by (4.1)),

$$
\lim _{\varepsilon \rightarrow 0} \int_{-\infty}^{\infty} \varphi(x) \int_{-\infty}^{\infty} \gamma(\varepsilon a) h(a, x) e^{-i a \theta(x)} d a d x
$$

exists for every fine function $\varphi$. Also

$$
w_{\varepsilon}(x)=\int_{-\infty}^{\infty} \gamma(\varepsilon a) h(a, x) e^{-i a \theta(x)} d a
$$


is an infinitely differentiable function of $x \in \mathbb{R}$ for each value of $\varepsilon$ which runs through the points of an interval of $\mathbb{R}$. In fact, let $x_{0} \in \mathbb{R}$ and choose $a<b$ such that $x_{0} \in(a, b)$. By Definition 3.1, for every $a<b$ there exists a constant $C$ such that

$$
\left|\int_{-\infty}^{\infty} \gamma(\varepsilon a) h(a, x) e^{-i a \theta(x)} d a\right| \leq C \int_{-\infty}^{\infty}|\gamma(\varepsilon a)|(1+|a|)^{N} d a
$$

for every $\varepsilon, x \in[a, b]$ and the integral on the right of the above inequality is finite (and independent of $x$ ). Consequently from [5, Theorem 11.2] there exists a weak function which equals the weak limit $\lim _{\varepsilon \rightarrow 0} w_{\varepsilon}(x)$ for $x \in \mathbb{R}$.

\section{Some properties of the transform}

In this section, we obtain the weak derivative of the weak function defined in Theorem 1 and we also note a property of the above weak function. Accordingly, we state

Theorem 2. If $h \in H$ and $\theta: \mathbb{R} \rightarrow \mathbb{R}$ is an infinitely differentiable function such that $\theta^{\prime}(x) \neq 0$ for every $x \in \mathbb{R}$, then

$$
\frac{d}{d x} \int_{-\infty}^{\infty} h(a, x) e^{-i a \theta(x)} d a=\int_{-\infty}^{\infty}\left\{\frac{\partial}{\partial x} h(a, x)-i a \theta^{\prime}(x) h(a, x)\right\} e^{-i a \theta(x)} d a
$$

for $x \in \mathbb{R}$, where the derivative on the left-hand side is a weak one.

Proof. By Theorem 1 and [5, Theorem 11.4], we have that the left-hand side of (5.1) equals

$$
\lim _{\varepsilon \rightarrow 0} \frac{d}{d x} \int_{-\infty}^{\infty} \gamma(\varepsilon \alpha) h(\alpha, x) e^{-i a \theta(x)} d a
$$

for $x \in \mathbb{R}$ (where $\gamma$ is a good function such that $\gamma(0)=1$ ). But, using the same arguments as for (4.7), the above weak limit is, in fact,

$$
\lim _{\varepsilon \rightarrow 0} \int_{-\infty}^{\infty} \gamma(\varepsilon a)\left\{\frac{\partial}{\partial x} h(a, x)-i a \theta^{\prime}(x) h(a, x)\right\} e^{-i a \theta(x)} d a
$$

which exists for $x \in \mathbb{R}$ (since $\frac{\partial h}{\partial x}-i a \theta^{\prime}(x) h \in H(N+1, c, d)$ ) and is denoted by the right-hand side of (5.1).

Theorem 3. With the notation of Theorem 2, it holds that for every $x \in \mathbb{R}$

$$
\int_{-\infty}^{\infty} \frac{\partial}{\partial a} h(a, x) e^{-i a \theta(x)} d a=\int_{-\infty}^{\infty} i \theta(x) h(a, x) e^{-i a \theta(x)} d a
$$

Proof. If $\gamma$ is a good function, an integration by parts gives

$$
\begin{aligned}
\lim _{\varepsilon \rightarrow 0} \int_{-\infty}^{\infty} \gamma(\varepsilon a) \frac{\partial}{\partial a}\left\{h(a, x) e^{-i a \theta(x)}\right\} d a \\
\quad=\lim _{\varepsilon \rightarrow 0}\left\{-\int_{-\infty}^{\infty} \frac{\partial}{\partial a} \gamma(\varepsilon a) h(a, x) e^{-i a \theta(x)} d a\right\} .
\end{aligned}
$$

It holds that

$$
\lim _{\varepsilon \rightarrow 0} P(\varepsilon)=0
$$

where

$$
P(\varepsilon)=\int_{-\infty}^{\infty} \varphi(x) \int_{-\infty}^{\infty} \frac{\partial}{\partial a} \gamma(\varepsilon a) h(a, x) e^{-i a \theta(x)} d a d x
$$


and $\varphi$ is any fine function, for the following reason.

We substitute $\varepsilon a$ by $y$ in the integrand of $P$ and we consider $\varepsilon$ outside the two integral signs. Then by (4.1), $P$ is $F$ with $\gamma^{\prime}$ instead of $\gamma$ and by Theorem $1, P$ is equal to a finite number of integrals of the form (4.3) and (4.5) with $\gamma^{\prime}$ in place $\gamma$, the limits of which as $\varepsilon \rightarrow 0$ are zero.

Therefore, the weak limit on the left-hand side of (5.2) exists and is equal to zero. Hence, by Theorem 1, we conclude that

$$
\int_{-\infty}^{\infty} \frac{\partial}{\partial a}\left\{h(a, x) e^{-i a \theta(x)}\right\} d a=0
$$

and the theorem is proved.

\section{Weak limits}

The following result is useful in calculating the weak functions defined in Theorem 1.

Theorem 4. Let $\theta: \mathbb{R} \rightarrow \mathbb{R}$ be an infinitely differentiable function with $\theta^{\prime}(x) \neq 0$ for every $x \in \mathbb{R}$, and $\left(h_{n}\right)$ a sequence such that $h_{n}: \mathbb{R} \times \mathbb{R} \rightarrow \mathbb{R}$ and the following three conditions hold:

(i) $h_{n} \in(N, c, d)$ for every $n \in \mathbb{N}$;

(ii) there exist $h \in H(N, c, d)$ and $a_{0} \in \mathbb{R}$ such that

$$
\lim _{n \rightarrow \infty} h_{n}\left(a_{0}, x\right)=h\left(a_{0}, x\right)
$$

for every $x \in \mathbb{R}$

(iii) for every $p \in \mathbb{N}$ the sequence $\left(\frac{\partial^{p}}{\partial a^{p}} h_{n}\right)$ converges uniformly on $\mathbb{R}$ as $n \rightarrow \infty$. Then

$$
\lim _{n \rightarrow \infty} \int_{-\infty}^{\infty} h_{n}(a, x) e^{-i a \theta(x)} d a=\int_{-\infty}^{\infty} h(a, x) e^{-i a \theta(x)} d a .
$$

Proof. From (4.6), with $h_{n}$ instead of $h$, we obtain that for every fine $\varphi$,

$$
\lim _{\varepsilon \rightarrow 0} \int_{-\infty}^{\infty} \varphi(x) \int_{-\infty}^{\infty} \gamma(\varepsilon a) h_{n}(a, x) e^{-i a \theta(x)} d a d x=K(n),
$$

where $K(n)$ is the right-hand side of (3.7) with 1 instead of $\gamma(\varepsilon a)$ and $h_{n}$ in place of $h$. Therefore, by Theorem 1 , we arrive at

$$
\lim _{n \rightarrow 0} \int_{-\infty}^{\infty} \varphi(x) \int_{-\infty}^{\infty} h_{n}(a, x) e^{-i a \theta(x)} d a d x=\lim _{n \rightarrow \infty} K(n)
$$

Let $K_{0}(n)$ up to $K_{5}(n)$ denote the integrals $I_{0}$ up to $I_{5}$, respectively, of Remark 3.14 with 1 instead of $\gamma(\varepsilon a)$ and $h_{n}$ instead of $h$. We observe that the inner integrals of $K_{0}(n), K_{1}(n)$, and $K_{2}(n)$ are bounded by

$$
\int_{-\infty}^{\infty}(1+|a|)^{N-c v} d a
$$

when $c+d \leq 1$, and by

$$
\int_{-\infty}^{\infty}(1+|a|)^{N+d v-v} d a
$$

when $c+d>1$, which are finite integrals since we have chosen a suitable $v$ such that $N-c v<-1$ and $N+d v-v<-1$, respectively, for each case. Therefore the integrals $K_{0}(n), K_{1}(n)$, and $K_{2}(n)$ converge uniformly on $\mathbb{N}$. The same holds for the integrals 
$K_{3}(n), K_{4}(n)$ and $K_{5}(n)$. Hence we can take the limit as $n \rightarrow \infty$ under both integral signs, in order to calculate their limits.

It can be proved by induction that for every $p=0,1,2, \ldots$,

$$
\frac{\partial^{p+\lambda}}{\partial x^{p} \partial a^{\lambda}} h_{n}(a, x) e^{-i a \theta(x)}=\sum_{q=0}^{p}(-1)^{q}\left(\begin{array}{l}
p \\
q
\end{array}\right) \frac{\partial^{p-q}}{\partial x^{p-q}}\left\{\frac{\partial^{\lambda}}{\partial a^{\lambda}} h_{n}(a, x) \frac{\partial^{q}}{\partial x^{q}} e^{-i a \theta(x)}\right\} .
$$

Consequently, by also using Leibniz's Theorem for the derivative of an integral [1] (since the conditions for the exchange of derivative and integral are fulfilled by continuity and uniform convergence) and integrating by parts $j-i-r$ times in $K_{1}$ and $q-m-s$ times in $K_{4}$, we obtain

$$
\begin{aligned}
K_{1}(n)= & \sum_{r=0}^{j-i}(-1)^{j-i}\left(\begin{array}{c}
j-i \\
r
\end{array}\right) \int_{-\infty}^{\infty} \psi^{(j-i-r)}(x) \int_{-\infty}^{\infty}\left\{\left\{\frac{1-\chi(a)}{a}\right\}^{\mu}\right\}^{(v-\tau-\mu)} \\
& \times \frac{\partial^{\tau}}{\partial a^{\tau}} h_{n}(a, x) \frac{\partial^{r}}{\partial x^{r}} e^{-i a \theta(x)} d a d x
\end{aligned}
$$

and

$$
\begin{aligned}
K_{4}(n)= & \sum_{s=0}^{q-m}(-1)^{q-m}\left(\begin{array}{c}
q-m \\
s
\end{array}\right) \int_{-\infty}^{\infty} \omega^{(q-m-s)}(x) \\
& \times \int_{-\infty}^{\infty} \chi(a)\left\{\left\{\frac{1-\chi(a)}{a}\right\}^{\rho}\right\}^{(\lambda-1-\sigma-\rho)} \frac{\partial^{\sigma}}{\partial a^{\sigma}} h_{n}(a, x) \frac{\partial^{s}}{\partial x^{s}} e^{-i a \theta(x)} d a d x
\end{aligned}
$$

where the fine functions

$$
\begin{gathered}
\psi(x)=\left\{\Theta_{1}^{\mu}(x) \Theta^{v-\mu}(x)\right\}^{(\mu-j)} \varphi^{(i)}(x), \\
\omega(x)=\{1-\theta(x) \Theta(x)\}\left\{\Theta_{1}^{\rho}(x) \Theta^{\lambda-1-\rho}(x)\right\}^{(\rho-q)} \varphi^{(m)}(x) .
\end{gathered}
$$

On account of the assumptions of the theorem (see also interchange of limit and derivative in [1]), we observe that the limits as $n \rightarrow \infty$ of the integrands of $K_{0}(n)$ up to $K_{5}(n)$ are obtained by replacing the function $\frac{\partial^{p} h_{n}}{\partial a^{p}}$ by $\frac{\partial^{p} h}{\partial a^{p}}$ with $p=0,1, \ldots, v$. Therefore because of (6.2), (6.3) and the definitions of $K_{0}(n), K_{2}(n), K_{3}(n), K_{5}(n)$, it follows that

$$
\lim _{n \rightarrow \infty} K(n)=G,
$$

where $G$ is defined by (4.6). Hence the left-hand side of (6.1) equals

$$
\lim _{\varepsilon \rightarrow 0} \int_{-\infty}^{\infty} \varphi(x) \int_{-\infty}^{\infty} \gamma(\varepsilon a) h(a, x) e^{-i a \theta(x)} d a d x
$$

for every fine $\varphi$. By using Theorem 1 we complete the proof.

\section{References}

1. R. G. Bartle, The Elements of Real Analysis, Wiley International Edition, New York, 1964.

2. H. Bremmer, Diffraction problems of microwave optics, I. R. E. Trans. on Antennas and Propagation AP-3 (1955), 222-228.

3. R. A. Handelsman and J. S. Lew, Asymptotic expansion of a class of integral transforms with algebraically dominated kernels, J. Math. Anal. Appl. 35 (1971), 405-433.

4. D. S. Jones, Generalized transforms and their asymptotic behaviour, Philos. Trans. Roy. Soc. London, Ser. A 265 (1969), 1-43.

5. - The Theory of Generalised Functions, Cambridge University Press, 1982. 
6. D. S. Jones and M. Kline, Asymptotic expansion of multiple integrals and the method of stationary phase, J. Math. Phys. 37 (1958), 1-28.

7. M. I. Kontorovich and U. K. Muravév, The derivation of the laws of reflection of geometrical optics on the basis of the asymptotic treatment of the diffraction problems, Jour. of Tech. Physics 22 (1952), 394-407.

8. J. Lavoine and O. P. Misra, Abelian theorems for the distributional Stieltjes transformation, Math. Proc. Camb. Phil. Soc. 86 (1979), 287-293.

9. M. J. Lighthill, Fourier Analysis and Generalized Functions, Cambridge University Press, New York, 1958.

10. A. Poulkou, Weak transforms and their limits, Bull. Greek Math. Soc. 24 (1983), 73-104.

11. W. Rudin, Functional Analysis, McGraw-Hill, New York, 1973.

12. L. Schwartz, Mathematics for Physical Sciences, Addison-Wesley, Reading, Mass., 1966.

13. Théorie des Distributions, Hermann, Paris. 1966.

University of Athens, Department of Mathematics, Panepistemiopolis, 15784 Athens, GREECE 